\title{
Comments
}

\section{Stereotactic and Functional Neurosurgery}

Stereotact Funct Neurosurg 2008;86:187

DOI: 10.1159/000126942

\section{Check the 'Active Ingredients' of Your Medications}

\author{
Comment on ‘Does Dopamine Still Have a Leading Role \\ in Advanced Parkinson's Disease after Subthalamic \\ Stimulation?' (Stereotact Funct Neurosurg 2008;86: \\ 184-186)
}

Michael Schulder

North Shore LIJ Health System, Manhasset, N.Y., USA

In this paper, the authors give a rationale for an extrastriatal dopaminergic pathway in Parkinson's disease (PD) based on a clinical observation. They describe a young patient who underwent successful placement of bilateral subthalamic nucleus (STN) deep brain stimulation electrodes (DBS). He had an excellent clinical response at first to stimulation, with improvement in UPDRS and dyskinesia scores while not taking any levodopa, although he did take the dopamine agonist cabergoline.

About 14 months after surgery the patient had an acute motor worsening. The only apparent reason for this was his taking an antihistamine to counter a contact allergy. One of the components of this drug was fluphenazine, a potent dopamine antagonist. Several days after stopping this medication the patient regained his previously functional postoperative status.

The authors suggest that since the STN is 'downstream' from the striatal feedback loops of the basal ganglia circuits as they are currently understood, a dopamine antagonist would not inhibit the benefits of STN DBS by an effect on the striatum. Rather, inhibition of extrastriatal dopaminergic pathways may have been to blame for this patient's decline after taking fluphenazine. The possibility remains that there was an inhibitory effect by fluphenazine on striatal dopaminergic circuits that were enhanced with cabergoline.

This is an interesting report that the authors reasonably propose as a spur to study alternate physiological pathways for PD. From a purely practical point of view, clinicians should be alert to the fact that commonly available antiallergic medications may contain dopamine antagonists that can worsen their patients' parkinsonian symptoms.

Michael Schulder, MD

North Shore University Hospital

300 Community Drive, 9 Tower Manhasset, NY 11030 (USA)

Tel. +1 516562 3065, Fax +1 5165622635

E-Mail schulder@nshs.edu
Stereotact Funct Neurosurg 2008;86:187-188

DOI: 10.1159/000126943

\section{DBS and Dopamine}

Comment on ‘Does Dopamine Still Have a Leading Role in Advanced Parkinson's Disease after Subthalamic Stimulation?' (Stereotact Funct Neurosurg 2008;86: 184-186)

Philip A. Starr

University of California San Francisco and Veteran's Affairs Medical Center, San Francisco, Calif., USA

How does DBS improve symptoms in PD? To a first approximation, the current concept is the following: As a downstream consequence of striatal dopamine denervation, basal ganglia output (GPi and its glutamatergic upstream partner, STN) have excessive and abnormally patterned activity. DBS of STN or GPi 'corrects' this abnormal activity, allowing thalamocortical pathways and/or brainstem structures receiving BG outflow to function more normally. As PD progresses, symptoms arise that are resistant to DBS (such as hypophonia, postural instability, and dementia) but these are also resistant to dopaminergic pharmacotherapy, and are thought to be due to involvement of non-dopaminergic pathways that also degenerate in $\mathrm{PD}$.

If this view is entirely correct, one might not expect further compromise of basal ganglia dopaminergic pathways to affect the ability of STN-DBS to suppress L-dopa-responsive cardinal PD symptoms. Hence the interest of this case report by Jabre et al. They report that a patient who had considerable benefit from STN DBS loses that benefit for 3 days following inadvertent ingestion of the D1, D2 antagonist fluphenzine. This finding is used to argue that STN DBS must act in part via a dopaminergic mechanism: either striatal dopamine, or the less dense but potentially important dopaminergic innervation of other BG nuclei, including a dopaminergic innervation of the STN itself.

Prior neurochemical studies of the effects of DBS on DA release in the basal ganglia are not entirely consistent. In humans, it has been shown that STN DBS does not alter striatal dopamine release [1]. However, in rat brain slices, STN DBS does result in depolarization of nigral dopaminergic cells (which could result in striatal DA release in intact animals) [2]. In intact parkinsonian rats chronically treated with L-dopa, STN DBS alters the responsiveness of striatal cells to L-dopa [3]. The current literature leaves open the possibility that STN DBS may act in part by a mechanism dependent on dopaminergic transmission, including extrastriatal dopaminergic transmission, but does not confirm this view resoundingly.

\section{KARGER}

Fax +41613061234 E-Mail karger@karger.ch www.karger.com
(C) 2008 S. Karger AG, Base

$1011-6125 / 08 / 0863-0187 \$ 24.50 / 0$ 